\title{
Nonacog Beta Pegol: A Review in Haemophilia B
}

\author{
Yahiya Y. Syed ${ }^{1}$
}

Published online: 9 November 2017

(C) Springer Nature 2017, corrected publication July/2018

\begin{abstract}
Nonacog beta pegol $\left[\right.$ Refixia $^{\circledR}$ (EU)] is an intravenously-administered, glycoPEGylated recombinant factor IX (FIX), with an extended terminal half-life. It is approved in the EU for the treatment and prophylaxis of bleeding in patients aged $\geq 12$ years with haemophilia B. The therapeutic efficacy and safety of nonacog beta pegol was demonstrated in the phase 3 Paradigm trials in previously treated adolescents and adults with haemophilia B. In Paradigm 2, nonacog beta pegol showed good haemostatic effects when treating bleeds on-demand, and reduced annualized bleeding rates when used as a once-weekly prophylaxis. It also improved some health-related quality of life measures in adult patients. The longer-term efficacy of nonacog beta pegol was demonstrated in the open-label extension Paradigm 4 trial. In Paradigm 3, nonacog beta pegol effectively maintained intraoperative and postoperative haemostasis. Nonacog beta pegol was well tolerated in phase 3 clinical trials in patients with haemophilia B, with no evidence of FIX inhibitor formation, allergic reactions or thromboembolic complications. In conclusion, nonacog beta pegol is effective and well tolerated in the on-demand, prophylaxis and perioperative
\end{abstract}

The original version of this article was revised due to a retrospective Open Access request.

The manuscript was reviewed by: $\boldsymbol{L}$. $\boldsymbol{N}$. Boggio, Rush Hemophilia and Thrombophilia Center, Rush University, Chicago (IL), USA; $\boldsymbol{G}$. Castaman, Center for Bleeding Disorders and Coagulation, Careggi University Hospital, Florence, Italy; $\boldsymbol{R}$. Klamroth, Department for Internal Medicine, Haemophilia Treatment Centre, Vivantes Klinikum im Friedrichshain, Berlin, Germany; J. N. Mahlangu, Haemophilia Comprehensive Care Centre, University of the Witwatersrand, Johannesburg, South Africa.

Yahiya Y. Syed

demail@springer.com

1 Springer, Private Bag 65901, Mairangi Bay, Auckland 0754, New Zealand settings in adolescent and adults with haemophilia B. Its extended half-life allows for once-weekly prophylaxis. Therefore, nonacog beta pegol is a useful additional treatment option for patients with haemophilia B.

Nonacog beta pegol: clinical considerations in haemophilia B

Intravenously-administered, glycoPEGylated recombinant FIX with an extended terminal half-life

Shows good haemostatic effects when treating bleeds on-demand

Once-weekly prophylaxis significantly reduces annualized bleeding rates, including in patients with target joints

Maintains intraoperative and postoperative haemostasis

Well tolerated, with no evidence of FIX inhibitor formation, allergic reactions or thromboembolic complications in phase 3 clinical trials

\section{Introduction}

Haemophilia B is a rare X-linked recessive bleeding disorder caused by a deficiency of coagulation factor IX (FIX), resulting from mutations in the FIX gene [1]. The severity of bleeding in haemophilia B is correlated with the residual endogenous FIX levels, based on which, the disease severity is classified as mild, moderate or severe (defined as FIX levels of $>0.05-0.40, \quad 0.01-0.05$ and $<0.01 \mathrm{IU} / \mathrm{ml}$, 
respectively; i.e. $>5$ to $<40 \%, 1-5 \%$ and $<1 \%$ of normal levels) $[1,2]$. The mild form is characterized by rare spontaneous bleeding and severe bleeding during major trauma or surgery, the moderate form by occasional spontaneous bleeding and prolonged bleeding during minor trauma or surgery, and the severe form by spontaneous bleeding into joints and muscles $[1,3]$. According to the CHESS study, approximately one-third of the haemophilia B population in Europe have the severe form, which is associated with a high annual treatment cost [4]. Depending on bleeding sites, haemophilia B can be serious (joints, muscles, mucous membranes) or life-threatening (intracranial, neck/throat, gastrointestinal) [1]. Thus, haemophilia B is associated with frequent long-term disability, impaired health-related quality of life (HR-QOL) and substantial treatment cost $[1,3,4]$.

Haemophilia B can be treated with intravenous FIX replacement therapy, administered on demand to reduce bleeding episodes and as prophylaxis to prevent bleeding and joint destruction, with the aim of preserving normal musculoskeletal function [1]. Currently, prophylaxis is considered to be the standard of care for haemophilia B in developed countries [5]. In this approach, plasma FIX levels are maintained above $0.01 \mathrm{IU} / \mathrm{mL}$ (1\%), thereby converting the disease severity phenotype from severe to moderate $[1,3,5,6]$; maintaining FIX levels $>1 \%$ may be useful in some patients [7]. Prophylaxis is associated with a few challenges, such as need for venous access, frequent FIX administrations, variable pharmacokinetics and increased initial cost; however, prophylaxis is considered superior to on-demand therapy as it prevents bleeding-related complications, particularly haemophilic arthropathy, in patients with severe disease $[3,5,6]$.

Highly purified FIX concentrates [plasma-derived FIX (pdFIX) and recombinant FIX (rFIX)] are routinely used for the treatment of haemophilia $\mathrm{B}[1,3]$. These preparations have showed haemostatic efficacy in on-demand, prophylaxis and perioperative settings and are associated with reduced thromboembolic complications relative to intermediate purity FIX concentrates, such as prothrombin complex concentrates (PCCs), which are rarely used now [3]. However, as pdFIX and rFIX have a relatively short plasma half-life $\left(\mathrm{t}_{1 / 2}\right.$; typically $16-19 \mathrm{~h}$ ), they require frequent administration (2-3 times weekly) to maintain protective prophylaxis FIX levels $[3,5,6]$. Therefore, current development efforts have focused on extending the $t_{1 / 2}$ of rFIX by modifying its physiological and pharmacokinetic properties with the aim of reducing treatment burden and thereby potentially improving treatment compliance and clinical outcomes [3, 5, 6]. Approaches to prolong the $t_{1 / 2}$ have included covalent attachment of a polyethylene glycol (PEG) molecule (i.e. glycoPEGylation) to the rFIX activation peptide [8] or protein fusion (fusing human albumin [9] or Ig [10] to rFIX).

Nonacog beta pegol [Refixia $\left.{ }^{\circledR}(\mathrm{EU})\right]$ is an intravenouslyadministered, purified, serum-free, glycoPEGylated rFIX
[11] that has been approved in the EU for the treatment and prophylaxis of bleeding in patients aged $\geq 12$ years with haemophilia B [12]. This narrative review discusses pharmacological, therapeutic efficacy and tolerability data relevant to the use of nonacog beta pegol in this patient population.

\section{Pharmacodynamic Properties of Nonacog Beta Pegol}

The rFIX molecule in nonacog beta pegol is produced in Chinese hamster ovary (CHO) $\mathrm{K} 1$ cells by recombinant DNA technology $[11,12]$. No additional human- or animalderived materials are used in the cell culture, purification, conjugation or formulation of nonacog beta pegol $[11,12]$. The chemical structure and the specific activity of the rFIX in nonacog beta pegol is generally similar to that of conventional pdFIX or rFIX [11]. Nonacog beta pegol is produced using an acceptor site-directed glycoPEGylation of rFIX; this technique is designed to overcome any loss in FIX activity and ensure normal regulation of the active form. In this technique, a 40-kDa PEG moiety is attached to one $(95 \%$, mono-PEGylated) or both $(5 \%$, di-PEGylated) $\mathrm{N}$-glycans within the activation peptide of rFIX; $\mathrm{N}$-glycans are located away from the functional catalytic domain of the FIX molecule and present only in the circulating zymogen form of FIX. During the coagulation process, the activation peptide with the attached PEG is cleaved off, leaving activated rFIX (rFIXa) [11].

In vitro, functional properties of nonacog beta pegol are not substantially affected by glycoPEGylation [11]. The PEG moiety did not affect the kinetics of nonacog beta pegol activation by factor XIa, while it decreased the activation by factor VIIa-tissue factor complex by $\approx 50 \%$, primarily because of an increased $K_{m}$. GlycoPEGylation did not affect binding of factor VIIIa to activated nonacog beta pegol or the kinetics of factor $\mathrm{X}$ activation by activated nonacog beta pegol. Similarly, nonacog beta pegol retained its ability to bind to endothelial cells (i.e. the ability to bind collagen IV associated with the vascular endothelium), although higher concentrations were required relative to unmodified $\mathrm{rFIX}$ [11].

The specific clotting activity of nonacog beta pegol was equivalent to that of unmodified rFIX, with no significant differences in clot parameters between the two seen in a thromboelastography analysis of human haemophilia B whole blood [11]. In vitro, nonacog beta pegol retained $73 \%$ of its specific activity relative to unmodified rFIX in plasma. In animal models, nonacog beta pegol exhibited increased in vivo recovery and $t_{1 / 2}$, and decreased clearance, relative to rFIX. Nonacog beta pegol showed haemostatic efficacy in mice and dog models of haemophilia B [11]. 


\section{Pharmacokinetic Properties of Nonacog Beta Pegol}

The pharmacokinetic profile of nonacog beta pegol has been characterised in previously treated adult, adolescent and paediatric patients with haemophilia $B$ enrolled in the Paradigm clinical programme [8, 12-18]. The pharmacokinetics of nonacog beta pegol are best described by a one-compartment model, with first-order elimination, according to a population pharmacokinetic model [16]. In adult patients, nonacog beta pegol is assumed to have dose linearity over a dose range of $25-100 \mathrm{U} / \mathrm{kg}$, based on the area under the FIX activity time curve (AUC) from the time of administration to infinity and FIX plasma activity 30 min after administration [13]. Dose-linear FIX activity was also observed in 10 and $40 \mathrm{IU} / \mathrm{kg}$ dosage groups in adolescents and adult patients [16]. Single-dose and steadystate pharmacokinetics of nonacog beta pegol $40 \mathrm{IU} / \mathrm{kg}$ in various age groups are summarized in Table 1 [16]. After nonacog beta pegol administration, FIX activity increased to normal levels and then decreased over time following a monoexponential decay [16]. Bodyweight-adjusted clearance of nonacog beta pegol was higher in adolescent

Table 1 Pharmacokinetics nonacog beta pegol $40 \mathrm{IU} / \mathrm{kg}$ in previously treated patients with haemophilia B [16]

\begin{tabular}{|c|c|c|c|}
\hline \multirow[t]{2}{*}{ Parameter } & \multirow{2}{*}{$\begin{array}{l}\text { Children } \\
\left(\leq 12 \text { years }{ }^{\mathrm{a}}\right) \\
\text { Single-dose } \\
(n=25)\end{array}$} & \multicolumn{2}{|c|}{$\begin{array}{l}\text { Adolescents and } \\
\text { adults }(\geq 13 \text { years })\end{array}$} \\
\hline & & $\begin{array}{l}\text { Single } \\
\text { dose } \\
(n=9)\end{array}$ & $\begin{array}{l}\text { Steady- } \\
\text { state }^{\mathrm{b}} \\
(n=9)\end{array}$ \\
\hline $\begin{array}{l}\text { FIX activity at } 30 \mathrm{~min} \\
\text { post dose }(\mathrm{IU} / \mathrm{mL})\end{array}$ & 0.57 & 0.83 & 1.02 \\
\hline $\begin{array}{l}\text { Incremental recovery at } \\
30 \mathrm{~min} \text { post dose }(\mathrm{U} / \mathrm{mL} \\
\text { per } \mathrm{U} / \mathrm{kg})\end{array}$ & 0.02 & 0.02 & 0.02 \\
\hline $\begin{array}{l}\text { AUC from time } 0-\infty \\
(\mathrm{IU} \cdot \mathrm{h} / \mathrm{mL})\end{array}$ & 51.1 & 86.9 & 141.3 \\
\hline $\begin{array}{l}\text { Volume of distribution at } \\
\text { steady state }(\mathrm{mL} / \mathrm{kg})\end{array}$ & 70.2 & 50.6 & 64.0 \\
\hline Accumulation ratio & 1.27 & 1.35 & 1.43 \\
\hline Clearance $(\mathrm{mL} / \mathrm{h} / \mathrm{kg})$ & 0.7 & 0.4 & 0.4 \\
\hline Mean residence time (h) & 100.3 & 118.3 & 153.4 \\
\hline Terminal half-life (h) & 73.0 & 85.1 & 110.8 \\
\hline $\begin{array}{l}\text { FIX activity } 168 \mathrm{~h} \text { post } \\
\text { dose (i.e. trough level) } \\
\text { (IU/mL) }\end{array}$ & 0.10 & 0.16 & 0.31 \\
\hline
\end{tabular}

Data are geometric mean values, measured based on plasma FIX activity using one-stage clotting assay

$A U C$ area under the FIX activity time curve, FIX factor IX

${ }^{a}$ Nonacog beta pegol is approved only in patients aged $\geq 12$ years

${ }^{\mathrm{b}}$ Following once-weekly administration patients than in adults. However, no dosage adjustment is required for adolescents (Sect. 6) [12].

The FIX activity (a surrogate endpoint of efficacy) profile of nonacog beta pegol supports its use in the prophylaxis, on-demand and perioperative settings $[8,13,14,16]$. Once-weekly prophylaxis with a $40 \mathrm{IU} / \mathrm{kg}$ dosage maintained high FIX activity $168 \mathrm{~h}$ post-dose (trough) in adolescents and adults (Table 1) [8, 16]. Where reported, the estimated mean trough activity with this regimen $(0.273 \mathrm{IU} / \mathrm{mL})$ was significantly $(p<0.001)$ above $0.01 \mathrm{IU} / \mathrm{mL}$ [8]. Similarly, following a single $40 \mathrm{IU} /$ $\mathrm{kg}$ dosage, the FIX activity $168 \mathrm{~h}$ post-dose was $\geq 0.10 \mathrm{IU} / \mathrm{mL}$ in children, adolescents and adults; following once weekly administration of this dosage, the mean FIX trough activity at steady state was $\geq 0.17 \mathrm{IU} /$ $\mathrm{mL}$ across all age groups [16]. Therefore, when nonacog beta pegol is used, routine monitoring of FIX activity levels for the purpose of dosage adjustment is not required [12]. These data are supported by a post hoc estimation of FIX activity in adults, showing that the time to 3 and $1 \%$ FIX activity (i.e. 0.03 and $0.01 \mathrm{IU} / \mathrm{mL}$ ) was 16.2 and 22.5 days following a dose normalized to $50 \mathrm{U} / \mathrm{kg}$ [13]. In patients undergoing a major surgery receiving a single preoperative bolus injection of $80 \mathrm{IU} / \mathrm{kg}$ in Paradigm 3 (Sect. 4.2), the median FIX activity increased from $0.27 \mathrm{IU} / \mathrm{mL}$ pre-dose to $1.43 \mathrm{IU} / \mathrm{mL} 30 \mathrm{~min}$ post-dose [14] and was 138,112 and $73 \%$ (i.e. $1.38,1.12$ and $0.73 \mathrm{IU} / \mathrm{mL}$ ) at 8,24 and $48 \mathrm{~h}$ post-dose, respectively [18].

Nonacog beta pegol showed a favourable pharmacokinetic profile relative to pdFIX or rFIX when the estimated means of selected pharmacokinetic parameters $\left(\mathrm{t}_{1 / 2}\right.$, incremental recovery, clearance, volume of distribution and AUC) were compared in adults [13]. The $t_{1 / 2}$ of nonacog beta pegol was 5.21and 4.80-fold higher than for pdFIX and rFIX (both $p<0.001)$. The incremental recovery of nonacog beta pegol was $94 \%(p<0.001)$ relative to rFIX, but it did not differ significantly relative to pdFIX [13]. These results are supported by a population pharmacokinetic model [17] of data from adults [13]. This model predicted that the mean steady-state trough levels with nonacog beta pegol $40 \mathrm{IU} / \mathrm{kg}$ once weekly prophylaxis would be approximately 8- and 4-times greater than with the standard prophylaxis regimens (40 IU/kg every 3 days) of pdFIX and rFIX, respectively [17]. The model also predicted reduced dosages, dosing frequency and total FIX consumption for nonacog beta pegol versus pdFIX or rFIX for the treatment of bleeds and perioperative haemostasis [17].

\section{Therapeutic Efficacy of Nonacog Beta Pegol}

The therapeutic efficacy of nonacog beta pegol in the prophylaxis and on-demand treatment of bleeding episodes in previously treated patients aged 13-70 years with 
haemophilia B was evaluated in the pivotal, randomized, single-blind, phase 3 Paradigm 2 trial [8]. The use of nonacog beta pegol in maintaining perioperative haemostasis in this patient population was investigated in the open-label, single-arm, phase 3 Paradigm 3 trial [14]. Patients completing either Paradigm 2 or Paradigm 3 trial were subsequently enrolled in the nonrandomized, openlabel extension Paradigm 4 trial [15]. The efficacy and safety of nonacog beta pegol was also assessed in previously treated paediatric patients aged 0-12 years in the phase 3 Paradigm 5 trial [19], which is briefly discussed in this review as nonacog beta pegol is not indicated for children aged $<12$ years in the EU (Sect. 6).

The Paradigm 2 [8] and Paradigm 3 [14] trials included males with haemophilia B (FIX activity $\leq 0.02 \mathrm{IU} / \mathrm{mL}$ ), with a history of $\geq 150$ exposure days to other FIX products. Exclusion criteria included current or historical presence of FIX inhibitors (i.e. FIX neutralizing IgG antibodies), HIV infection with a CD4 lymphocyte count of $\leq 200$ cells/ $\mu \mathrm{L}$ and a history of thromboembolic events [8, 14]. Paradigm 2 also excluded patients receiving immunomodulating or chemotherapeutic drugs and those with a platelet count of $<50,000 / \mu \mathrm{L}$, an ALT level of $>3$ times the upper limit of normal (ULN) or a creatinine level of $\geq 1.5$ times the ULN [8].

\subsection{Prophylaxis and On-demand Treatment}

In Paradigm 2, patients were randomized to prophylaxis with nonacog beta pegol 10 or $40 \mathrm{IU} / \mathrm{kg}$ once weekly for 52 weeks; an additional nonrandomized treatment group received on-demand nonacog beta pegol treatment for 28 weeks [8]. Patients could choose between prophylaxis and on-demand treatment. Bleeding episodes in all three treatment groups were treated with a single nonacog beta pegol dose of $40 \mathrm{IU} / \mathrm{kg}$ ( $80 \mathrm{IU} / \mathrm{kg}$ if bleeding was severe, i.e. intracranial, iliopsoas, neck or retroperitoneal bleeding). The main efficacy endpoints were the haemostatic effect when treating a bleeding episode, assessed by patient-rated haemostatic response as defined in Table 2, and the prophylactic effect, assessed by annualized bleeding rate (ABRs) during the trial (Table 3) [8]. In the EU, $10 \mathrm{IU} / \mathrm{kg}$ is below the recommended dosage of $40 \mathrm{IU} / \mathrm{kg}$ (Sect. 6), but is discussed in this review for comparison and completeness.

Paradigm 2 enrolled 74 patients (mean age 31.4 years), with 67 patients completing the trial, including 17 adolescents (13-17 years) and 50 adults (18-65 years) [8]. Of the 74 patients, 19 and $81 \%$ had moderate and severe haemophilia $\mathrm{B}$, respectively, and approximately one-half had previously received FIX products on-demand $(47 \%)$ or as prophylaxis $(53 \%)$. Among those who had received prophylaxis $(n=39), 54$ and $46 \%$ of patients had received rFIX and pdFIX, respectively. At baseline, $65 \%$ of patients had arthropathy and 54\% had target joints [8]. A target joint was defined, according to the European Paediatric Network for Haemophilia Management (PEDNET), as $\geq 3$ bleeds in a particular joint within a period of 6 months before trial, regardless of the causality of the bleed (i.e. spontaneous or traumatic) [8, 20].

Paradigm 4 was planned for an additional 50 exposure days to nonacog beta pegol to that received in Paradigm 2 [15]. It enrolled 71 patients, 66 from Paradigm 2 and five from Paradigm 3 (Sect. 4.2). Initially, Paradigm 4 had the same three treatment groups as in Paradigm 2: i.e. two prophylaxis groups (10 or $40 \mathrm{IU} / \mathrm{kg}$ once-weekly) and an on-demand group, with bleeds in all groups treated with a single $40 \mathrm{IU} / \mathrm{kg}$ ( $80 \mathrm{IU} / \mathrm{kg}$ for severe bleeds) dose. In addition, a third prophylaxis group (80 IU/kg every second week) was included following a late trial protocol amendment, although only two patients received this regimen, as the trial was stopped soon after the amendment. The patient and investigator selected the treatment group at trial entry and the patients were allowed to change treatment groups during the trial based on clinical manifestations and investigator's recommendations. At trial entry, 20 patients changed their treatment groups from their original group in Paradigm 2 and nine patients changed treatment groups during the trial [15].

Paradigm 5 enrolled 25 paediatric patients who received prophylaxis with nonacog beta pegol $40 \mathrm{IU} / \mathrm{kg}$ once weekly for 52 weeks during the trial [19]. Patients experiencing breakthrough bleeds received a single nonacog beta pegol dose of $40 \mathrm{IU} / \mathrm{kg}$ for mild to moderate bleeds or $80 \mathrm{IU} / \mathrm{kg}$ for severe bleeds [19].

\subsubsection{On-demand Treatment}

On-demand treatment with nonacog beta pegol $40 \mathrm{IU} / \mathrm{kg}$ (80 IU/kg for severe bleeding) was effective in treating bleeding episodes in patients with haemophilia $B$ $[8,15,19,20]$. Bleeding and haemostatic response data for all patients and individual treatment groups in the Paradigm 2 and 4 trials are summarized in Table 2 [8, 15]. In Paradigm 2, a total of 345 bleeding episodes (202 in the prophylaxis groups and 143 in the on-demand group) were treated with nonacog beta pegol, with an estimated successful haemostatic response rate of $92.2 \%$ (95\% CI 86.9-95.4) [8]. The success rate was the highest in the $40 \mathrm{IU} / \mathrm{kg}$ prophylaxis group, with $99 \%$ of bleeding episodes stopped with one injection (Table 2) [8]. There was one severe bleeding episode (in the knee joint) in the $10 \mathrm{IU} / \mathrm{kg}$ group, which was treated with nonacog beta pegol $80 \mathrm{IU} / \mathrm{kg}$, with the haemostatic response rated as excellent [8]. In Paradigm 4, a total of 207 bleeding episodes were treated with nonacog beta pegol, with a successful haemostatic response rate of $\approx 95 \%$ (Table 2) [15]. No severe bleeding episodes were reported during the trial [15]. 
Table 2 Efficacy of on-demand treatment with nonacog beta pegol in previously treated patients with haemophilia B in phase 3 trials

\begin{tabular}{|c|c|c|c|c|c|c|c|c|}
\hline \multirow[t]{2}{*}{ Treatment } & \multirow{2}{*}{$\begin{array}{l}\text { No. of } \\
\text { pts }^{\mathrm{a}}\end{array}$} & \multirow{2}{*}{$\begin{array}{l}\text { Pts with } \\
\text { bleeds }(\%)^{\mathrm{b}}\end{array}$} & \multirow{2}{*}{$\begin{array}{l}\text { No. of } \\
\text { bleeds }\end{array}$} & \multicolumn{3}{|c|}{ Type/location of bleeds (\%) } & \multirow{2}{*}{$\begin{array}{l}\text { Bleeds requiring } 1,2,3, \geq 4 \\
\text { injections to treat }(\%)\end{array}$} & \multirow{2}{*}{$\begin{array}{l}\text { Successful } \\
\operatorname{HSR}^{\mathrm{c}}(\%)\end{array}$} \\
\hline & & & & Spontaneous & Traumatic & Joint & & \\
\hline \multicolumn{9}{|l|}{ Paradigm 2 [8] } \\
\hline PRX $10 \mathrm{IU} / \mathrm{kg} \mathrm{qw}^{\mathrm{d}}$ & 30 & 83.3 & 132 & 68.9 & 29.5 & 81.1 & $84.1,12.1,1.5,2.3$ & 86.9 \\
\hline PRX 40 IU/kg qw ${ }^{\mathrm{d}}$ & 29 & 55.2 & 70 & 48.6 & 51.4 & 78.3 & $98.6,0,1.4,0$ & 97.1 \\
\hline On-demand & 15 & 93.3 & 143 & 71.3 & 28.7 & 76.4 & $83.9,14.0,0,2.1$ & 95.1 \\
\hline Total & 74 & 74.3 & 345 & 65.8 & 33.6 & 78.5 & $87.0,10.4,0.9,1.7$ & $92.4^{\mathrm{e}}$ \\
\hline \multicolumn{9}{|l|}{ Paradigm 4 [15] } \\
\hline PRX $10 \mathrm{IU} / \mathrm{kg}$ qw & 21 & 66.7 & 35 & 71.4 & 28.6 & 74.3 & $82.9,14.3,2.9,0$ & 97.1 \\
\hline PRX 40 IU/kg qw & 52 & 59.6 & 98 & 36.7 & 57.1 & 73.5 & $84.7,10.2,4.1,1.0$ & 94.8 \\
\hline PRX $80 \mathrm{IU} / \mathrm{kg}$ q2w & 2 & 50.0 & 1 & 0 & 100.0 & 100.0 & $100.0,0,0,0$ & 100.0 \\
\hline On-demand & 5 & 100.0 & 73 & 93.2 & 6.8 & 79.5 & $94.5,5.5,0,0$ & 93.1 \\
\hline Total & 71 & 69.0 & 207 & 62.3 & 34.8 & 75.8 & $87.9,9.2,2.4,0.5$ & 94.6 \\
\hline
\end{tabular}

See text for trial design details

$H S R$ haemostatic response, $P R X$ prophylaxis, pts patients, $q w$ once weekly, $q 2 w$ every second week

${ }^{\mathrm{a}}$ Number of pts randomized/assigned (Paradigm 2) or treated at any time during the trial (Paradigm 4)

${ }^{\mathrm{b}}$ In Paradigm 4, numbers are based on the treatment group at the time of each bleeding episode

${ }^{\mathrm{c}} \mathrm{HSR}$ was rated by the patients as follows: excellent $=$ abrupt pain relief and/or clear improvement in objective signs of bleeding within $8 \mathrm{~h}$ after a single injection; good $=$ noticeable pain relief and/or improvement in signs of bleeding within $8 \mathrm{~h}$ after a single injection; moderate $=$ probable or slight beneficial effect within the first $8 \mathrm{~h}$ after the first injection but requiring more than a single injection within $8 \mathrm{~h}$; poor $=$ either no improvement or worsening of symptoms within $8 \mathrm{~h}$ after 2 injections. Successful HSR was defined as an excellent or good HSR while a moderate or poor HSR was considered as failed HSR. Bleeding episodes with missing HSR were not included in the calculation

${ }^{\mathrm{d}}$ Randomized treatment groups

${ }^{e}$ Observed rate, which is slightly higher than the estimated rate reported in text

According to a post hoc analysis of Paradigm 2, nonacog beta pegol was effective in treating target joint bleeds [20]. At baseline, 13 and 15 patients in the 10 and $40 \mathrm{IU} / \mathrm{kg}$ prophylaxis groups had at least one target joint. During the trial, there were 49 and 19 breakthrough target joint bleeds requiring treatment, respectively, with 78 and $100 \%$ of these bleeds resolving with one nonacog beta pegol $40 \mathrm{IU} /$ $\mathrm{kg}$ injection. The successful haemostatic response rate was 81 and $95 \%$ in the 10 and $40 \mathrm{IU} / \mathrm{kg}$ groups [20].

In Paradigm 5, 42 breakthrough bleeds occurred in 15 of $25(60 \%)$ patients [19]. In these patients, on-demand treatment was associated with an overall successful haemostatic response rate of $92.9 \%$, with $86 \%$ of bleeds resolving with one injection [19].

\subsubsection{Prophylaxis}

In Paradigm 2, nonacog beta pegol $40 \mathrm{IU} / \mathrm{kg}$ once weekly prophylaxis effectively reduced ABRs in patients with haemophilia $B[8,20]$. In patients not receiving prophylaxis (i.e. on-demand treatment group) the median ABR was 15.58 [8], whereas it was 1.04 and 2.93 in the 40 and $10 \mathrm{IU} / \mathrm{kg}$ prophylaxis groups (Table 3) [8]. Although both prophylaxis regimens reduced the estimated mean ABRs, the predefined criteria for the effective prophylaxis in the overall population was met only in the $40 \mathrm{IU} / \mathrm{kg}$ group but not in the $10 \mathrm{IU} / \mathrm{kg}$ group (Table 3). FIX activity levels were sustained throughout the prophylaxis dosing intervals; the estimated mean spontaneous ABRs were only slightly higher towards the end of the intervals ( 1.87 during the first 4 days and 2.16 after 4 days of the previous administration) [8]. The prophylactic efficacy was also evident with respect to the proportion of patients who did not have a bleeding requiring treatment: 45 and $17 \%$ in the 40 and $10 \mathrm{IU} / \mathrm{kg}$ prophylaxis groups (Table 3 ), compared with $7 \%$ in the on-demand group [8].

Consistent with the overall population, in a subgroup of patients who entered the Paradigm 2 trial from a previous prophylaxis regimen, the prophylactic effect of nonacog beta pegol was more pronounced in the $40 \mathrm{IU} / \mathrm{kg}$ group (the estimated mean ABR decreased from 7.49 in the 12 months prior the trial to 3.33 during the trial) than in the $10 \mathrm{IU} / \mathrm{kg}$ group (5.13 to 4.68) [8]. In patients who entered the trial from previous on-demand treatment, the corresponding changes were 21.2 to 1.3 in the $40 \mathrm{IU} / \mathrm{kg}$ group and 17.9 to 4.3 in the $10 \mathrm{IU} / \mathrm{kg}$ group [8].

In Paradigm 2, the $40 \mathrm{IU} / \mathrm{kg}$ regimen was also more effective than the $10 \mathrm{IU} / \mathrm{kg}$ regimen in patients with target joints $[8,20]$. Using the PEDNET definition of a target joint, 15 and 13 patients in the 40 and $10 \mathrm{IU} / \mathrm{kg}$ prophylaxis 
Table 3 Prophylactic efficacy of nonacog beta pegol in previously treated patients with haemophilia B in phase 3 trials

\begin{tabular}{|c|c|c|c|c|c|c|c|}
\hline \multirow[t]{2}{*}{ Treatment } & \multirow{2}{*}{$\begin{array}{l}\text { No. of } \\
\mathrm{pts}^{\mathrm{a}}\end{array}$} & \multirow{2}{*}{$\begin{array}{l}\text { No } \\
\text { bleeds } \\
(\% \text { pts })\end{array}$} & \multicolumn{5}{|c|}{ Annualized bleeding rates } \\
\hline & & & Statistic & Spontaneous & Traumatic & Joint & Total \\
\hline \multicolumn{8}{|l|}{ Paradigm 2 [8] } \\
\hline \multirow[t]{2}{*}{$10 \mathrm{IU} / \mathrm{kg}$ qw } & 30 & 16.7 & Median (IQR) & $0.97(0.00-4.01)$ & $0.98(0.00-1.93)$ & NA & $2.93(0.99-6.02)$ \\
\hline & & & $\operatorname{Mean}^{\mathrm{b}}(95 \% \mathrm{CI})$ & $3.14(1.78-5.56)$ & $1.35(0.81-2.24)$ & NA & $4.56(3.01-6.90)$ \\
\hline \multirow[t]{2}{*}{$40 \mathrm{IU} / \mathrm{kg}$ qw } & 29 & 44.8 & Median (IQR) & $0.00(0.00-0.98)$ & $0.00(0.00-2.04)$ & $0.97(0.00-2.07)^{\mathrm{c}}$ & $1.04(0.00-4.00)$ \\
\hline & & & $\operatorname{Mean}^{\mathrm{b}}(95 \% \mathrm{CI})$ & $1.22(0.48-3.10)$ & $1.29(0.76-2.19)$ & NA & $2.51 *(1.42-4.43)^{\mathrm{d}}$ \\
\hline \multicolumn{8}{|l|}{ Paradigm 4 [15] } \\
\hline \multirow[t]{2}{*}{$10 \mathrm{IU} / \mathrm{kg} \mathrm{qw}$} & 20 & 30.0 & Median (IQR) & $1.05(0.00-2.16)$ & $0.00(0.00-1.01)$ & $0.97(0.00-1.41)$ & $1.36(0.00-2.23)$ \\
\hline & & & $\operatorname{Mean}^{\mathrm{b}}(95 \% \mathrm{CI})$ & $1.31(0.63-2.73)$ & $0.53(0.20-1.39)$ & $1.36(0.56-3.33)$ & $1.84(1.00-3.38)$ \\
\hline \multirow[t]{2}{*}{$40 \mathrm{IU} / \mathrm{kg}$ qw } & 49 & 40.8 & Median (IQR) & $0.00(0.00-1.00)$ & $0.00(0.00-1.10)$ & $0.00(0.00-1.97)$ & $1.00(0.00-2.03)$ \\
\hline & & & $\operatorname{Mean}^{\mathrm{b}}(95 \% \mathrm{CI})$ & $0.71(0.38-1.33)$ & $1.01(0.64-1.57)$ & $1.49(0.87-2.54)$ & $1.84(1.26-2.70)$ \\
\hline \multirow[t]{2}{*}{ Total } & 66 & 34.8 & Median (IQR) & $0.00(0.00-1.29)$ & $0.00(0.00-1.06)$ & $0.59(0.00-1.89)$ & $1.05(0.00-2.20)$ \\
\hline & & & $\operatorname{Mean}^{\mathrm{b}}(95 \% \mathrm{CI})$ & $0.89(0.55-1.42)$ & $0.87(0.57-1.32)$ & $1.46(0.91-2.34)$ & $1.84(1.33-2.56)$ \\
\hline
\end{tabular}

See text for trial design details

$I Q R$ interquartile range, $N A$ not available, $q w$ once weekly, $p t(s)$ patient(s)

$* p=0.01$ based on 1 -sided test of the null hypothesis that the estimated rate is at least 4.8 , evaluated at the $2.5 \%$ level

${ }^{a}$ Randomized pts (Paradigm 2) or pts who were on same treatment regimen for $\geq 3$ months (Paradigm 4)

${ }^{\mathrm{b}}$ Estimate of mean based on a Poisson regression model

${ }^{c}$ Data from the EU summary of product characteristics [12]

${ }^{\mathrm{d}}$ Prophylactic effect was confirmed based on a predefined criteria (i.e. the upper limit of the $95 \%$ CI was below 4.8, which corresponds to a $>60 \%$ reduction in a literature-based value of 12 bleeds/pt/year in pts receiving on-demand treatment)

groups had at least one target joint at trial entry, with each group having 24 target joints among them. Of these patients, 66.7 and $7.7 \%$ of patients in the 40 and $10 \mathrm{IU} / \mathrm{kg}$ groups did not have a bleeding requiring treatment in their target joints during the trial $[8,20]$. Furthermore, in the post hoc analysis, 71 and $29 \%$ of target joints at baseline in the respective groups had no bleeding during the trial [20]. The median ABR [interquartile range (IQR)] in target joints during the trial was $0.0(0.00-2.01)$ in the $40 \mathrm{IU} / \mathrm{kg}$ group and $2.59(1.85-5.22)$ the $10 \mathrm{IU} / \mathrm{kg}$ group [20]. The corresponding estimated mean ABRs (95\% CI) were 1.32 (0.35-5.05) and $4.10(2.59-6.51)$, respectively [20]. The prophylactic efficacy of the $40 \mathrm{IU} / \mathrm{kg}$ regimen in patients with target joints was further supported by the post hoc analysis [20] when the most recent definition of a target joint from the International Society on Thrombosis and Haemostasis [21] was used.

Paradigm 4 results showed that long-term prophylaxis with nonacog beta pegol remained effective in patients with haemophilia B [15]. Among patients who remained on the same treatment group for $\geq 3$ months $(n=66), 125$ bleeds were reported in 43 patients. The median ABR in these patients was $\approx 1$ (Table 3 ). In Paradigm 4 , ABRs in the $40 \mathrm{IU} / \mathrm{kg}$ group were low and similar to that seen in Paradigm 2. However, the difference between the 40 and $10 \mathrm{IU} / \mathrm{kg}$ groups in $\mathrm{ABR}$ and the number of patients without bleeds was diminished in Paradigm 4 relative to
Paradigm 2 (Table 3), which may be attributed to the differences in the trial designs (Sect. 4.1) [15].

Twelve patients had low estimated mean ABRs (1.26) with the nonacog beta pegol $10 \mathrm{IU} / \mathrm{kg}$ regimen in Paradigm 2 and continued this regimen in Paradigm 4 during which the ABR slightly increased to 1.97 [15]. Fourteen patients had high estimated mean ABRs (6.89) with the $10 \mathrm{IU} / \mathrm{kg}$ regimen in Paradigm 2 and switched to the $40 \mathrm{IU} / \mathrm{kg}$ regimen in Paradigm 4; in these patients, ABR decreased to 2.78 during Paradigm 4. In 22 patients who remained on the $40 \mathrm{IU} / \mathrm{kg}$ regimen throughout both trials, the estimated mean ABRs were 2.29 and 1.16 during Paradigm 2 and Paradigm 4, respectively [15].

In Paradigm 5, the median ABR was 1.0 (IQR 0.00-2.06) and the median spontaneous ABR was 0.00 in children receiving nonacog beta pegol prophylaxis $[12,19]$.

\subsubsection{Health-Related Quality of Life}

Nonacog beta pegol $40 \mathrm{IU} / \mathrm{kg}$ once-weekly prophylaxis was associated with improvements in HR-QOL in patients with haemophilia $\mathrm{B}$, as assessed by patient-reported outcome questionnaires in Paradigm 2 and 4 [8, 22]. In Paradigm 2, adult patients ( $\geq 17$ years of age) receiving the $40 \mathrm{IU} / \mathrm{kg}$ regimen had significant $(p \leq 0.031)$ improvements from baseline in Haemophilia Quality Of 
Life Questionnaire For Adults (Haem-A-QoL) total score and scores for certain domains therein (sport, feeling, partnership) at the end of trial; no significant changes were seen in the $10 \mathrm{IU} / \mathrm{kg}$ prophylaxis or on-demand treatment groups [22]. In Paradigm 4, adult patients who switched from 10 to $40 \mathrm{IU} / \mathrm{kg}$ group had significant $(p=0.016)$ improvements from baseline in Haem-A-QoL total scores and physical health domain score. In adolescent patients (13-16 years of age) receiving nonacog beta pegol prophylaxis, there were no significant changes from baseline in HR-QOL parameters as assessed by the Haemophilia Quality Of Life Questionnaire For Children (Haemo-QOL) III, according to limited data from Paradigm $2(n=15)$ and Paradigm $4(n=12)$ [22].

In Paradigm 2, patients receiving $40 \mathrm{IU} / \mathrm{kg}$ prophylaxis reported a significant $(p=0.030)$ improvement from baseline in their global health status on a EQ-5D visual analogue scale, while there was no significant change in the $10 \mathrm{IU} / \mathrm{kg}$ prophylaxis or on-demand treatment groups [22]. Patients reported on EQ-5D that mobility and pain/discomfort as the main problems over the course of Paradigm 2. In the $40 \mathrm{IU} / \mathrm{kg}$ group, the proportion of patients having 'some problem' with mobility decreased from $51.7 \%$ at baseline to $20.7 \%$ at the end of the trial. The corresponding change in patients with 'moderate or extreme' pain/discomfort was 44.8 to $27.6 \%$. Nonacog beta pegol was also associated with a significant $(p=0.016)$ improvement from baseline in the three-level version of the EQ-5D index score (using the UK value set) in the overall population in Paradigm 2, with no significant differences observed in individual treatment groups [22].

\subsection{Perioperative Management}

Paradigm 3 enrolled 13 patients with haemophilia B scheduled for a major surgery (nine orthopaedic, three dental and one gastrointestinal procedures), including seven patients transferred from Paradigm 2 or Paradigm 4 and six new patients naive to nonacog beta pegol [14].

At trial entry, naive patients received two $40 \mathrm{IU} / \mathrm{kg}$ dosages (separated by $4-8$ days) to assess hypersensitivity or anaphylactic reactions and to calculate in vivo recovery; transferred patients who had been receiving $10 \mathrm{IU} / \mathrm{kg}$ once weekly or on-demand treatment regimen received a single $40 \mathrm{IU} / \mathrm{kg}$ dose to calculate in vivo recovery, while recovery data for patients who had been receiving $40 \mathrm{IU} / \mathrm{kg}$ onceweekly was obtained from the preceding trial [14]. During the preoperative period, patients coming from a prophylaxis regimen received $40 \mathrm{IU} / \mathrm{kg}$ once weekly while those coming from an on-demand regimen received $40 \mathrm{IU} / \mathrm{kg}$ every 7-14 days. Preoperative treatment was continued for 2-8 weeks (naive patients) or 2-4 weeks (transferred patients). On the day of surgery ( $\geq 7$ days from the last dose of nonacog beta pegol), all patients received a single bolus injection $80 \mathrm{IU} / \mathrm{kg}$ within $4 \mathrm{~h}$ prior to the procedure. Postoperatively, fixed doses of $40 \mathrm{IU} / \mathrm{kg}$ were administered at the discretion of the investigator to maintain FIX levels of $0.4-0.6,0.3-0.5$ and $0.2-0.4 \mathrm{IU} / \mathrm{mL}$ during days $1-3$, 4-6 and 7-14, respectively [14].

In Paradigm 3, preoperative prophylaxis followed by a single presurgical nonacog beta pegol dose of $80 \mathrm{IU} / \mathrm{kg}$ was effective in maintaining the intraoperative haemostasis in patients undergoing a major surgery [14]. In all 13 cases, this outcome was rated as excellent (10 cases) or good (3 cases) by the investigator/surgeon, according to a predefined response scale. None of the patients required additional doses on the day of surgery. A median of two and three injections were administered during postoperative days 1-6 and 1-13, respectively. The mean total consumption of nonacog beta pegol during and after surgery was $241.2 \mathrm{IU} / \mathrm{kg}$ [14].

\section{Tolerability of Nonacog Beta Pegol}

Nonacog beta pegol was well tolerated in previously treated patients with haemophilia B in the phase 3 Paradigm clinical trials discussed in Sect. 4 [8, 14, 15, 19]. None of the patients withdrew treatment in these trials because of an adverse event $[8,14,15,19]$. The mean number of exposure days to nonacog beta pegol per patient was 54 in the prophylaxis and 14 in the on-demand groups in Paradigm 2 [8], with additional 54 and 16 days, respectively, in Paradigm 4 [15]. The mean number of exposure days was 63.6 in Paradigm 5 [19].

\subsection{General Tolerability Profile}

In Paradigm 2, 215 adverse events were reported in $81 \%$ of patients; however, the majority of these events were mild (183 events) or moderate ( 25 events) in severity, with only 7 events rated as severe [8]. The most common adverse events were nasopharyngitis ( $13.5 \%$ of patients; 13 events), influenza $(10.8 \% ; 10)$ and upper respiratory tract infection $(10.8 \% ; 10)$. Four serious adverse events (hip fracture, worsening of skin ulcer, retroperitoneal hematoma and abdominal pain) occurred in four patients (5.4\%) and were deemed unlikely to be related to nonacog beta pegol by the investigator [8].

The tolerability profile of nonacog beta pegol in the longer-term Paradigm 4 trial [15] was generally similar to that in Paradigm 2 [8]. In Paradigm 4, four adverse events (injection site rash, two occurrences of overdose in one patient and neutropenia) in three patients were deemed by the investigator as possibly or probably related to nonacog beta pegol. The injection site reaction did not reoccur and the case of neutropenia was mild and resolved by the end of the trial. 
The overdose was associated with high FIX activity levels, but the patient recovered without complications [15].

Physical examinations and clinical laboratory tests did not reveal any safety concerns with nonacog beta pegol in Paradigm 2-5 clinical trials $[8,14,15,19]$.

\subsection{Adverse Events of Special Interest}

FIX inhibitors (assessed by a Nijmegen-modified Bethesda assay) were not detected across Paradigm trials 1-5 (primary safety objective in Paradigm 2, 4 and 5 trials) [8, 13-15, 19]. Transient, low-titre, noninhibitory anti-nonacog beta pegol binding antibodies were detected in three patients (two were positive before exposure) in Paradigm 2 [8] and two patients in Paradigm 4 [15] receiving prophylaxis regimens. These antibodies had no effect on drug recovery and bleeding patterns [8]; they did not cross-react to rFIX and did not have any other effect of antibodies [15]. In Paradigm 2, one patient was positive for antibodies against $\mathrm{CHO}$ cell proteins before and after exposure to nonacog beta pegol [8]. In Paradigm 4, two patients developed low-titre antibodies against $\mathrm{CHO}$ cell proteins [15]; in two additional patients who had these antibodies before nonacog beta pegol treatment, titre levels were either reduced or unaffected by treatment [15].

In the phase 3 Paradigm trials, where reported, there were no thromboembolic events $[8,14,15,19]$ or allergic reactions $[8,19]$ and deaths $[8,14,19]$ related to nonacog beta pegol. One patient in the phase 1 Paradigm 1 trial had a severe hypersensitivity reaction probably related to nonacog beta pegol; this patient was treated for the reaction and fully recovered $7.5 \mathrm{~h}$ after the onset of the reaction [13].

\section{Dosage and Administration of Nonacog Beta Pegol}

Nonacog beta pegol is approved in the EU for the treatment and prophylaxis of bleeding in patients aged $\geq 12$ years with haemophilia B [12]. Its efficacy and tolerability have not yet been established in previously untreated patients. The recommended dosage for prophylaxis is $40 \mathrm{IU} / \mathrm{kg}$ once weekly; the dosage and administration intervals may be adjusted based on FIX levels achieved and bleeding tendency in individual patients. For on-demand treatment, a single $40 \mathrm{IU} / \mathrm{kg}$ dosage is recommended for early or more extensive haemarthrosis, muscle bleeding, oral bleeding or haematoma; for severe or life threatening haemorrhages, $80 \mathrm{IU} / \mathrm{kg}$ is recommended, but additional $40 \mathrm{IU} / \mathrm{kg}$ dosages can be administered. For perioperative haemostasis, the dosage level and administration intervals depend on the procedure and local practice. A single preoperative dosage of $40 \mathrm{IU} / \mathrm{kg}$ is recommended for minor surgery including tooth extraction (additional doses can be given if required).
The recommended regimen for major surgery is a single preoperative dosage of $80 \mathrm{IU} / \mathrm{kg}$, two postoperative dosage of $40 \mathrm{IU} / \mathrm{kg}$ at $1-3$ days intervals and $40 \mathrm{IU} / \mathrm{kg}$ once weekly after the first week until bleeding stops and healing is achieved. The dosage recommendations in adolescents (12-18 years) are the same as for adults [12].

Routine monitoring of FIX activity is not required when nonacog beta pegol is used (Sect. 3) [12]. When monitoring is needed, a chromogenic assay (e.g. Rox Factor IX or Biophen) is recommended over the one-stage clotting assay because of the interference of PEG with various activated partial thromboplastin time (aPTT) reagents in the latter [12]. If chromogenic assay is not possible, a one-stage clotting assay with an aPTT reagent qualified for use with nonacog beta pegol (e.g. Cephascreen ${ }^{\circledR}$ ) is recommended. A one-stage clotting assay using silica-based reagents is not recommended because nonacog beta pegol FIX activity is overestimated by $>400 \%$ with such reagents [12, 23]. Local prescribing information should be consulted for detailed information on the use of monitoring assays/reagents, special warnings and precautions, use in special patient populations, and reconstitution and administration procedures.

\section{Place of Nonacog Beta Pegol in the Management of Haemophilia B}

The World Federation of Hemophilia [1] and the European Association for Haemophilia and Associated Disorders [24] recommend a multidisciplinary comprehensive care for haemophilia, with the primary goal of prevention and treatment bleeding episodes with the deficient clotting factor [1]. Bleeding in patients with haemophilia B is managed by life-long FIX replacement therapy, administered on-demand, as routine prophylaxis or perioperatively, with treatment being individualised to the requirements of each patient $[1,3,25,26]$.

FIX concentrates are the treatment of choice for haemophilia $\mathrm{B}$, and whenever possible, pdFIX and rFIX are preferable over PCCs [1]. However, the use of conventional pdFIX and rFIX is limited by their relatively short half-life and thus typically require twice weekly dosing to maintain prophylactic FIX levels (Sect. 1). Consequently, rFIX products with extended half-life have been developed to reduce dosages and/or dosing frequency without a loss of haemostatic efficacy.

Nonacog beta pegol is a glycoPEGylated, extended halflife rFIX approved in the EU for use in patients aged $\geq 12$ years with haemophilia B [12]. It is a highly purified serum-free product, with no additional human- or animal-derived materials (Sect. 2). While the specific activity of nonacog beta pegol is equivalent to that of conventional pdFIX or rFIX (Sect. 2), nonacog beta pegol 
has fivefold longer $t_{1 / 2}$ than pdFIX or rFIX and has significantly greater incremental recovery than rFIX (Sect. 3). Once-weekly injections of nonacog beta pegol $40 \mathrm{IU} / \mathrm{kg}$ produced high FIX trough activity in patients with haemophilia B, with the estimated mean trough activities being significantly above the generally accepted protective prophylaxis level $(0.01 \mathrm{IU} / \mathrm{mL})$ (Sect. 3).

Nonacog beta pegol was effective in on-demand, prophylaxis and perioperative settings in previously treated adolescents and adults with haemophilia B in multinational phase 3 trials (Sect. 4). On-demand treatment with a 40 IU/ $\mathrm{kg}$ (80 IU/kg for severe bleeding) dosage was associated with a high successful haemostatic response rate, with the majority of bleeds resolving with one injection (Sect. 4.1.1). Prophylaxis with a $40 \mathrm{IU} / \mathrm{kg}$ once-weekly regimen met the predefined criteria for effective prophylaxis (a $>60 \%$ reduction in $A B R$ relative to historical values), with the efficacy confirmed in the longer-term (Sect. 4.1.2). Nonacog beta pegol was also effective in on-demand treatment and prophylaxis in patients with target joints (Sect. 4.1). The prophylactic regimen was associated with improvements in HR-QOL in adult patients (Sect. 4.1.3). In patients with haemophilia B undergoing a major surgery, perioperative administration of nonacog beta pegol effectively maintained intraoperative and postoperative haemostasis (Sect. 4.2).

Nonacog beta pegol was well tolerated in previously treated adolescents and adults with haemophilia B in clinical trials (Sect. 5). Development of FIX inhibitors is the most serious complication of FIX replacement therapy and is associated with allergic reactions and reduced efficacy $[1,3,24]$. No FIX inhibitors or allergic reactions were reported in nonacog beta pegol recipients in phase 3 trials (Sect. 5.2). PEGylated proteins are considered less immunogenic than unmodified proteins [3], which may have contributed to the absence of FIX inhibitors with nonacog beta pegol. However, nonacog beta pegol trials included patients previously treated with FIX who did not develop FIX inhibitors (Sect. 4), while the risk of FIX inhibitor formation is increased in previously untreated patients with haemophilia B [3]. The safety and efficacy of nonacog beta pegol in previously untreated children is being investigated in the ongoing Paradigm 6 trial (NCT02141074). In addition to FIX inhibitors, there is a potential risk of thromboembolic complications with FIX therapy and the risk is higher with low purity preparations [12]. No thromboembolic events were reported in nonacog beta pegol recipients in clinical trials, although patients with a history of thromboembolic events were excluded in these trials.

Nonacog beta pegol is the first rFIX preparation to use glycoPEGylation half-life extension technology. The metabolism and excretion of PEG is driven largely by the absolute PEG load and its molecular weight [27]. PEG is cleared via glomerular filtration and high molecular weight
PEGs $(>30 \mathrm{kDa})$ are not efficiently filtered, resulting in potential accumulation in the lysosome [27]. However, in rats, radiolabelled nonacog beta pegol was eliminated via the kidneys and liver, with PEG-related radioactivity detected in both urine and faeces, indicating that these clearance pathways may contribute to the disposition kinetics of PEG moiety [28]. Although PEGylated protein therapies are generally well tolerated in humans [29], considering the life-long nature of FIX therapy, the safety of chronic PEG exposure needs ongoing surveillance in patients receiving nonacog beta pegol $[3,13]$.

In addition to nonacog beta pegol, two other rFIX products with an extended half-life (albutrepenonacog alfa [30] and eftrenonacog alfa [31]) have been approved in the $\mathrm{EU}$ and elsewhere for use in haemophilia B. While nonacog beta pegol is indicated only for patients aged $\geq 12$ years, the other two products are indicated for all age groups. A potential advantage with nonacog beta pegol is that monitoring of FIX activity is not required post administration (Sect. 3); FIX activity monitoring is advised during the course of treatment with albutrepenonacog alfa and eftrenonacog alfa to guide the dose and dosing frequency. Comparative efficacy, safety and pharmacoeconomics data for these products are required to define their relative positioning in the management of haemophilia $\mathrm{B}$.

In conclusion, nonacog beta pegol is effective and well tolerated in the on-demand, prophylaxis and perioperative settings in adolescents and adults with haemophilia B. The prolonged $t_{1 / 2}$ of nonacog beta pegol allows for onceweekly prophylaxis, which reduces the treatment burden relative to pdFIX and rFIX products with standard halflives. Nonacog beta pegol is therefore a useful additional treatment option for patients with haemophilia B.

Data Selection Nonacog Beta Pegol: 86 records identified

$$
\text { Duplicates removed } 21
$$

Excluded at initial screening (e.g. press releases; news reports; not relevant drug/indication)

Excluded during initial selection (e.g. preclinical study; reviews; case reports; not randomized trial)

Excluded during writing (e.g. reviews; duplicate data; small patient number; nonrandomized/phase I/II trials)

Cited efficacy/tolerability articles Cited articles not efficacy/tolerability

Search Strategy: EMBASE, MEDLINE and PubMed from 1946 to present. Clinical trial registries/databases and websites were also searched for relevant data. Key words were R Nonacog beta pegol, Refixia, N9-GP, haemophilia B, hemophilia B, factor IX, FIX, deficient, deficiency. Records were limited to those in English language. Searches last updated 27 October 2017 
Acknowledgements During the peer review process, the manufacturer of nonacog beta pegol was also offered an opportunity to review this article. Changes resulting from comments received were made on the basis of scientific and editorial merit

\section{Compliance with Ethical Standards}

Funding The preparation of this review was not supported by any external funding.

Conflict of interest Yahiya Syed is a salaried employee of Adis/ Springer, is responsible for the article content and declares no relevant conflicts of interest.

Additional information about this Adis Drug Review can be found at http://www.medengine.com/Redeem/72FBF0602C81013B.

Open Access This article is distributed under the terms of the Creative Commons Attribution-NonCommercial 4.0 International License (http://creativecommons.org/licenses/by-nc/4.0/), which permits any noncommercial use, duplication, adaptation, distribution and reproduction in any medium or format, as long as you give appropriate credit to the original author(s) and the source, provide a link to the Creative Commons license and indicate if changes were made.

\section{References}

1. Srivastava A, Brewer AK, Mauser-Bunschoten EP, et al. Guidelines for the management of hemophilia 2nd edition. 2013. http://www.wfh.org. Accessed 14 Aug 2017.

2. White GC II, Rosendaal F, Aldedort LM, et al. Definitions in hemophilia. Recommendation of the scientific subcommittee on factor VIII and factor IX of the scientific and standardization committee of the International Society on Thrombosis and Haemostasis. Thromb Haemost. 2001;85(3):560.

3. Nazeef M, Sheehan JP. New developments in the management of moderate-to-severe hemophilia B. J Blood Med. 2016;7:27-38.

4. O'Hara J, Hughes D, Camp C, et al. The cost of severe haemophilia in Europe: the CHESS study. Orphanet $\mathrm{J}$ Rare Dis. 2017;12(1):106.

5. Peyvandi F, Garagiola I, Biguzzi E. Advances in the treatment of bleeding disorders. J Thromb Haemost. 2016;14(11):2095-106.

6. Peyvandi F, Garagiola I, Young G. The past and future of haemophilia: diagnosis, treatments, and its complications. Lancet. 2016;388(10040):187-97.

7. Skinner MW. WFH: closing the global gap: achieving optimal care. Haemophilia. 2012;18(Suppl. 4):1-12.

8. Collins PW, Young G, Knobe K, et al. Recombinant long-acting glycoPEGylated factor IX in hemophilia B: a multinational randomized phase 3 trial. Blood. 2014;124(26):3880-6.

9. Santagostino E, Martinowitz U, Lissitchkov T, et al. Long-acting recombinant coagulation factor IX albumin fusion protein (rIXFP) in hemophilia B: results of a phase 3 trial. Blood. 2016;127(14):1761-9.

10. Powell JS, Pasi KJ, Ragni MV, et al. Phase 3 study of recombinant factor IX Fc fusion protein in hemophilia B. N Engl J Med. 2013;369(24):2313-23.

11. Østergaard H, Bjelke JR, Hansen L, et al. Prolonged half-life and preserved enzymatic properties of factor IX selectively PEGylated on native $\mathrm{N}$-glycans in the activation peptide. Blood. 2011;118(8):2333-41.

12. Nonacog beta pegol (Refixia ${ }^{\circledR}$ ): summary of product characteristics. 2017. http://www.ema.europa.eu. Accessed 14 Aug 2017.
13. Negrier C, Knobe K, Tiede A, et al. Enhanced pharmacokinetic properties of a glycoPEGylated recombinant factor IX: a first human dose trial in patients with hemophilia B. Blood. 2011;118(10):2695-701.

14. Escobar MA, Tehranchi R, Karim FA, et al. Low-factor consumption for major surgery in haemophilia $\mathrm{B}$ with long-acting recombinant glycoPEGylated factor IX. Haemophilia. 2017;23(1):67-76.

15. Young G, Collins PW, Colberg T, et al. Nonacog beta pegol (N9GP) in haemophilia B: a multinational phase III safety and efficacy extension trial (Paradigm 4). Thromb Res. 2016;141:69-76.

16. Tiede A, Abdul-Karim F, Carcao M, et al. Pharmacokinetics of a novel extended half-life glycoPEGylated factor IX, nonacog beta pegol (N9-GP) in previously treated patients with haemophilia B: results from two phase 3 clinical trials. Haemophilia. 2017;23(4):547-55.

17. Collins PW, Moss J, Knobe K, et al. Population pharmacokinetic modeling for dose setting of nonacog beta pegol (N9-GP), a glycoPEGylated recombinant factor IX. J Thromb Haemost. 2012;10(11):2305-12.

18. Rebinyn ${ }^{\circledR}$ (Coagulation Factor IX (Recombinant), GlycoPEGylated) lyophilized powder for solution for intravenous injection: US prescribing information. 2017. https://www.fda.gov. Accessed 11 Sept 2017.

19. Carcao M, Zak M, Abdul Karim F, et al. Nonacog beta pegol in previously treated children with hemophilia B: results from an international open-label phase 3 trial. J Thromb Haemost. 2016;14(8):1521-9.

20. Negrier C, Young G, Abdul Karim F, et al. Recombinant longacting glycoPEGylated factor IX (nonacog beta pegol) in haemophilia B: assessment of target joints in multinational phase 3 clinical trials. Haemophilia. 2016;22(4):507-13.

21. Blanchette VS, Key NS, Ljung LR, et al. Definitions in hemophilia: communication from the SSC of the ISTH. J Thromb Haemost. 2014;12(11):1935-9.

22. Chowdary P, Kearney S, Regnault A, et al. Improvement in healthrelated quality of life in patients with haemophilia B treated with nonacog beta pegol, a new extended half-life recombinant FIX product. Haemophilia. 2016;22(4):e267-74.

23. Rosen P, Rosen S, Ezban M, et al. Overestimation of N-glycoPEGylated factor IX activity in a one-stage factor IX clotting assay owing to silica-mediated premature conversion to activated factor IX. J Thromb Haemost. 2016;14(7):1420-7.

24. Colvin BT, Astermark J, Fischer K, et al. European principles of haemophilia care. Haemophilia. 2008;14(2):361-74.

25. Makris M. Prophylaxis in haemophilia should be life-long. Blood Transfus. 2012;10(2):165-8.

26. Sorensen B, Auerswald G, Benson G, et al. Rationale for individualizing haemophilia care. Blood Coagul Fibrinolysis. 2015;26(8):849-57.

27. Baumann A, Tuerck D, Prabhu S, et al. Pharmacokinetics, metabolism and distribution of PEGs and PEGylated proteins: quo vadis? Drug Discov Today. 2014;19(10):1623-31.

28. Sternebring O, Christensen JK, Bjornsdottir I. Pharmacokinetics, tissue distribution, excretion, and metabolite profiling of PEGylated rFIX (nonacog beta pegol, N9-GP) in rats. Eur J Pharm Sci. 2016;92:163-72.

29. Webster R, Elliott V, Park BK, et al. PEG and PEG conjugates toxicity: towards an understanding of the toxicity of PEG and its relevance to PEGylated biologicals. In: Veronese FM, editor. PEGylated protein drugs: basic science and clinical applications. Basel: Birkhäuser Basel; 2009. p. 127-46.

30. Lyseng-Williamson KA. Coagulation factor IX (recombinant), albumin fusion protein (albutrepenonacog alfa; Idelvion ${ }^{\circledR}$ ): a review of its use in haemophilia B. Drugs. 2017;77(1):97-106.

31. Hoy SM. Eftrenonacog alfa: a review in haemophilia B. Drugs. 2017;77(11):1235-46. 